\title{
A controlled distributed parameter model for a fluid-flexible structure system: numerical simulations and experiment validations
}

\author{
Bogdan Robu; Lucie Baudouin and Christophe Prieur
}

\begin{abstract}
We consider the problem of active reduction of vibrations in a fluid-flexible structure system. In the aerospace domain, we are actually interested in the system that couples the deflection of a plane wing and the sloshing of the fuel inside the wing's tank. The control is performed using piezoelectric patches and the main difficulty comes from the complex coupling between the flexible modes of the wing and the sloshing modes of the fuel. We establish an infinite-dimensional model for the global system and then a finite-dimensional approximation calculated using the first modes and validated on the experimental setup. A feedback controller is used to show the effectiveness of the closed loop in attenuating vibrations.

Index Terms-PDE experiment, fluid-flexible structure, pole placement
\end{abstract}

\section{INTRODUCTION}

Smart structures occupied a major place in the control research area during the last two decades. They are now used for their capability of attenuating the vibrations and measuring the deformation of structure. For instance consider recent feedback control techniques devoted to the active vibration reduction of flexible structures see [1], [6].

In the applicative domain of aerospace, we study the general problem of the suppression of a plane wing's vibration considering that the wing is in interaction with the movement of the fuel inside the wing's tank. Recent developments in the aerospace applications lead to more and more flexible wings. The actual system we study (see Figure 1) is an example of a coupled fluid-flexible structure system. Some studies investigate the use of piezoelectric patches to effectively suppress the vibrations. However, only a few results are already available in the literature for fluid-structure systems. One can see e.g. [13] for a recent theoretical result and [16] for a validation of the method by means of experimental results. There are less studies of fluid-structure system dedicated to aerospace applications but one can read [15] where controllers are designed using a numerical model.

The first aim of this paper is to derive an infinite dimensional model of the specific fluid-(flexible)structure system. The distribution of entries and the number of degrees of freedom of possible applications motivate us to consider a model written using partial differential equations. The difficulties that we have to manage are twofold. On the one hand the flexible structure is comparable to a plate, and we have two independent space variables (in contrast to the classical cantilever beam which does not have any width).

CNRS ; LAAS ; 7 avenue du colonel Roche, F-31077 Toulouse, France Université de Toulouse ; UPS, INSA, INP, ISAE ; LAAS ; F-31077 Toulouse, France brobullaas.fr, baudouin@laas.fr, cprieurelaas.fr

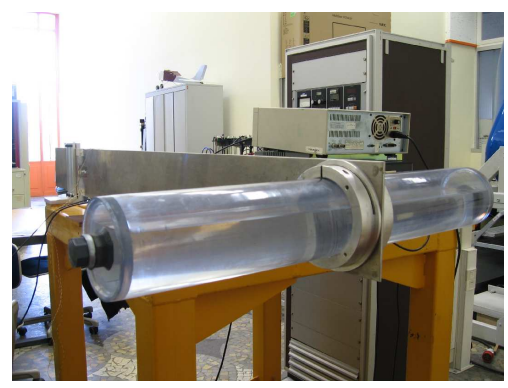

Fig. 1. Plant description: the rectangular plate and the horizontal cylinder

The study of flexible plates has been already considered in the literature (see [14]) but they are usually based on finitedimensional model. On the other hand, the interaction of the flexible plate with the fluid asks to introduce a dynamical fluid model which is also an infinite-dimensional equation and which is coupled with the plate equation.

The first contribution of this paper is therefore the computation of an infinite-dimensional model for the fluid-structure system. Then we compute a stabilizing controller which is based on a pole-placement feedback. We check up on simulations that there is no spillover effect when closing the loop. Finally we present some experiments on a real setup.

The paper is organized as follows. We present in Section II the plant under consideration. The plate is equipped with piezoelectric patches (sensors and actuators). Then in Section III we establish the fluid-structure model of our system. We compute in Section IV a simple stabilizing controller which is a pole-placement feedback control law. Section IV is also devoted to numerical simulations and to experiments on the real setup.

\section{Plant DESCRIPTION}

The system we want to control is located at ISAEENSICA, Toulouse, France and has been constructed to have the vibration frequencies of a real plane wing with fuel [15]. Our goal is to build a pole placement controller that will attenuate the vibrations of a plate despite the shaking of a tank filled with liquid and inserted at the end of the plate.

The device is composed of an aluminium rectangular plate and a plexiglas horizontal cylindrical tank filled with liquid (see Figure 1).

The plate has the length along the horizontal axis and the width along the vertical one; it is clamped on one side and free on the three others. The characteristics of the aluminium plate are given in Table I. 


\begin{tabular}{|c|c|c|}
\hline Plate length & $\mathrm{L}$ & $1.36 \mathrm{~m}$ \\
\hline Plate width & $\mathrm{l}$ & $0.16 \mathrm{~m}$ \\
\hline Plate thickness & $\mathrm{h}$ & $0.005 \mathrm{~m}$ \\
\hline Plate density & $\rho$ & $2970 \mathrm{~kg} \mathrm{~m}^{-3}$ \\
\hline Plate Young modulus & $\mathrm{Y}$ & $75 \mathrm{GPa}$ \\
\hline Plate Poisson coefficient & $\nu$ & 0.33 \\
\hline
\end{tabular}

TABLE I

PLATE CHARACTERISTICS

The piezoelectric actuators and sensors of the plant are made from PZT (lead zirconate titanate). They are glued next to the plate's clamped side. Two sensors are also located on the opposite side of the plate with respect to the actuators. The characteristics of the sensors and the actuators are given in Table II.

\begin{tabular}{|c|c|c|}
\hline Actuator length & $L_{\text {piezo }}$ & $0.14 \mathrm{~m}$ \\
\hline Actuator width & $l_{\text {piezo }}$ & $0.075 \mathrm{~m}$ \\
\hline Actuator thickness & $h_{\text {piezo }}$ & $0.0005 \mathrm{~m}$ \\
\hline Sensor length & $L_{\text {cpiezo }}$ & $0.015 \mathrm{~m}$ \\
\hline Sensor width & $l_{\text {cpiezo }}$ & $0.025 \mathrm{~m}$ \\
\hline Sensor thickness & $h_{\text {cpiezo }}$ & $0.0005 \mathrm{~m}$ \\
\hline Actuator/Sensor density & $\rho_{\text {piezo }}$ & $7800 \mathrm{~kg} \mathrm{~m}^{-3}$ \\
\hline Actuator/Sensor Young modulus & $Y_{\text {piezo }}$ & $67 \mathrm{GPa}$ \\
\hline Actuator piezoelectric coefficient & $d_{31}$ & $-210 e^{-12} \mathrm{~m} \mathrm{~V} \mathrm{~V}^{-1}$ \\
\hline Sensor piezoelectric coefficient & $e_{31}$ & $-9.6 \mathrm{~N}(\mathrm{Vm})^{-1}$ \\
\hline Actuator/Sensor Poisson coefficient & $\nu_{\text {piezo }}$ & 0.3 \\
\hline
\end{tabular}

TABLE II

CHARACTERISTICS OF THE PIEZOELECTRIC PATCHES

The tank is located at about $0.05 \%$ length of the plate's free side and is symetrically spread along the horizontal axis. Due to the configuration of the total system (cf Figures 1 and 2) we infer that the tank undergoes a longitudinal movement when the plate has a flexion movement and a pitch movement when the plate has a torsion movement. It has the dimensions given in Table III and it can be filled with water or ice up to an arbitrary level. If the tank is filled with ice we can easily model it by a steady mass. When the tank is filled with water but the water level is close to 0 or close to the cylinder diameter (tank empty or tank completely filled), as there is no sloshing behaviour, the modeling process is similar.

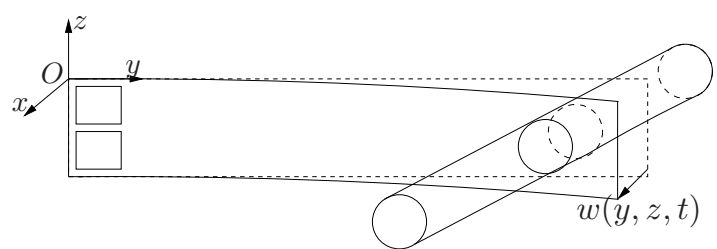

Fig. 2. Deformation of the rectangular plate

\begin{tabular}{|c|c|}
\hline Tank exterior diameter & $0.11 \mathrm{~m}$ \\
\hline Tank interior diameter & $0.105 \mathrm{~m}$ \\
\hline Tank length & $0.5 \mathrm{~m}$ \\
\hline Tank density & $1180 \mathrm{~kg} \mathrm{~m}^{-3}$ \\
\hline Tank young modulus & $4.5 \mathrm{GPa}$ \\
\hline \multicolumn{2}{|c|}{ TABLE III }
\end{tabular}

CHARACTERISTICS OF THE CYLINDRICAL TANK

\section{Modeling OF THE SYSTEM}

In this section we compute the mathematical model of the system previously presented. We first present an infinitedimensional model using operator matrices and then a finitedimensional model considering only some of the system's modes.

\section{A. Infinite-dimensional model}

On the one hand, considering the plate, we assume the kinematic hypothesis given by [2, Chapter 11.1]. This allows us to calculate the kinetic and potential energies as well as the mass and stiffness matrices. More details for calculations are given in [7, Chapter 4.6]. Using the mass and stiffness matrices one can infer the partial derivative equation of the plate:

$$
m_{s} \frac{\partial^{2} w}{\partial t^{2}}+\zeta(w) \frac{\partial w}{\partial t}+Y I_{s} \Delta^{2} w=\frac{\partial^{2} m_{y}}{\partial y^{2}}+\frac{\partial^{2} m_{z}}{\partial z^{2}}
$$

where $\zeta(w)$ is an operator quantifying the damping, $w=w(y, z, t)$ is the displacement, $m_{s}$ mass per unit plate area, $Y$ the Young modulus and $I_{s}=\frac{h^{3}}{12\left(1-\nu^{2}\right)}$ the moment of inertia of the plate. $\Delta$ is the Laplace operator with $\Delta^{2}$ being equal to $\left(\frac{\partial^{2}}{\partial y^{2}}+\frac{\partial^{2}}{\partial z^{2}}\right)^{2}$. The $m_{z}, m_{y}$ are external moments, along the $z$ and $y$-axis, delivered to the plate by the actuators (see [4] or [3]) and by the sloshing modes. Further on (1) is to be solved using the boundary conditions given in [2, Chapter 8.1.1] or [3].

Later in our study we are using the homogeneous partial derivative equation in the absence of external forces:

$$
\frac{m_{s}}{Y I_{s}} \frac{\partial^{2} w}{\partial t^{2}}+\Delta^{2} w=0
$$

The displacement $w$ will then be expressed in a Hilbertian base using Ritz functions:

$w(y, z, t)=\sum_{k=1}^{\infty} \eta_{k}(y, z) q_{k}(t)=\sum_{k=1}^{\infty} Y_{i_{k}}(y) Z_{j_{k}}(z) q_{k}(t)$

where $Y_{i_{k}}(y)$ and $Z_{j_{k}}(z)$ are the Ritz functions of two beams along the $y$-axis and $z$-axis. For a more detailled description of the choice of Ritz functions see [7, Chapter 5.2].

On the other hand, we now consider the longitudinal movement of the liquid along the $x$-axis. Because the liquid motion is starting from rest, there is a velocity potential $\phi(x, y, z, t)$ such that (see [12, Chapter 1.12]) the equation of liquid continuity is written

$$
\frac{\partial^{2} \phi}{\partial x^{2}}+\frac{\partial^{2} \phi}{\partial y^{2}}+\frac{\partial^{2} \phi}{\partial z^{2}}=0
$$

and the linearized equation of liquid motion is

$$
\frac{\partial \phi}{\partial t}+\frac{p}{\rho}+g(z-h)-C_{0} x=0
$$

where $C_{0}$ stands for the acceleration along the $x$-axis, $g$ for the gravitational acceleration and $h$ for the liquid height in the container at rest position; $\rho$ and $p$ are the density and the pressure of the liquid. Further on (4) and (5) are to be solved using the boundary conditions detailled in [12, Chapter 1.9]. 
As in [5, Chapter 1.2], we can give the expression of the oscillating free surface $z$ as:

$$
z=h+\delta(x, y, t)
$$

where $\delta(x, y, t)$ is the small displacement of the free surface above the undisturbed level $z=h$.

One can notice from [9, Chapters 1 and 2] that the solution of the sloshing problem depends on the geometry of the tank. In the case of horizontal cylindrical tank, as it is our case, the difficulty resides in the fact that the shape of the horizontal cylinder does not fit into any standard coordinate system and thus the velocity potential, which is quantifying the sloshing, cannot be derived using separation variable method. In our case (horizontal cylindrical tank that undergoes longitudinal movement) the longitudinal mode frequencies are simply curves faired through experimental data as are no analytical results available (see [5, Chapter 1.6]).

The solution we propose to overcome this difficulty is to make a geometrical approximation, the cylindrical tank being approximated by a rectangular one with the same sloshing frequencies but for which there are analytical results for the calculation of modes and forces/moments. In order to calculate the parameters of the rectangular "virtual" tank we use the following approach: we choose the length of the rectangular tank equal to the one of the cylindrical one, the width of the rectangular tank equal to the width of the free surface of the liquid and the height of the rectangular tank so that the liquid volume in both containers is the same. After comparing the sloshing frequencies of the rectangular tank to the ones calculated from the experimental data we observe that the difference is only $10^{-2} \mathrm{~Hz}$.

Further on $\phi(x, y, z, t)$ and $\delta(x, y, t)$ are calculated by solving (4), (5) and (6) with proper boundary conditions (see [10] for a rectangular tank of length $a$ along the $x$-axis and width $b$ along the $y$-axis). As the movement of the liquid is along the $x$-axis it can be proven that the free surface displacement and the velocity potential are not depending on $y$ :

$$
\begin{aligned}
\delta(x, y, t) & =\delta(x, t)=\sum_{i=0}^{\infty} r_{i}(t) \cos (\Xi i x) \\
\phi(x, y, z, t) & =\phi(x, z, t) \\
& =\sum_{i=0}^{\infty} \dot{r}_{i}(t) \frac{\cosh (\Xi i z)}{\Xi i \sinh \left(k_{i} h\right)} \cos (\Xi i x)
\end{aligned}
$$

where $\Xi i=\pi \frac{i}{a}$ and $\dot{r_{i}}=\frac{d r_{i}}{d t}$. By replacing (6), (7) and (8) in (5) we get a differential equation of $r_{i}$ which is to be solved for the free surface. This allow us to compute the $r_{i}$ functions. After tedious calculations we have the total moment exerted by the liquid sloshing:

$$
\begin{aligned}
m_{y}= & -2 \rho b \sum_{i=1,3,5, \ldots}^{\infty} \frac{\ddot{r}_{i}(t)}{\Xi i^{3}}\left[\frac{1}{\tanh (\Xi i h)}+\frac{2}{\sinh (\Xi i h)}\right] \\
& +\frac{\rho C_{0} a^{3} b}{12}+2 \rho b \sum_{i=1,3,5, \ldots}^{\infty} \frac{\ddot{r}_{i}(t)}{\Xi i^{3}} \frac{h \Xi i}{2}
\end{aligned}
$$

Further on, for the coupling, (9) is used in the moment expression $m_{y}$ of (1) along with the moment delivered by the actuators. More explanations are given for the finitedimensional case in paragraph III-B.3.

\section{B. Finite-dimensional approximation}

Knowing that the influence of system's modes is inversely proportional to the mode's frequency we legitimate that we may approximate the displacement by the first $N$ modes of the system. We are detailing in this subsection the calculation of the finite-dimensional approximation of our model. We aim at describing a state space model where the state vector should gather the first $N$ vibration modes of the plate and the first $N$ sloshing modes of the tank.

1) Plate model: In this section we are calculating the finite-dimensional approximation of (1) for the rectangular plate.

We write the finite-dimensional approximation for the clamped-free-free-free plate in the absence of the tank using equation (1). The approximation of the effect of the tank will be considered in Section III-B.3.b. We write

$$
\left\{\begin{array}{c}
\dot{X}_{p}(t)=A_{p} X_{p}(t)+B_{p} u(t) \\
y(t)=C_{p} X_{p}(t)
\end{array}\right.
$$

where $X_{p}=\left(\begin{array}{lllll}\dot{q}_{1} & \omega_{1} q_{1} & \cdots & q_{N} & \omega_{N} q_{N}\end{array}\right)$ - the $q_{k}$ being the same as in (3) - is the state space vector, and the dynamic matrix $A_{p}$ is written as:

$$
A_{p}=\left(\begin{array}{cccc}
A_{p_{1}} & 0 & \cdots & 0 \\
0 & A_{p_{2}} & \cdots & 0 \\
& \cdots & & \\
0 & 0 & \cdots & A_{p_{N}}
\end{array}\right)
$$

with $A_{p_{k}}=\left(\begin{array}{cc}-2 \zeta_{k} \omega_{k} & -\omega_{k} \\ \omega_{k} & 0\end{array}\right)$ for every $k$ from 1 to $N$.

The frequency of the $k^{t h}$ mode is $\omega_{k}$ in $\left[\mathrm{rad} \mathrm{s} s^{-1}\right]$ given in [2, Chapter 11] and $\zeta_{p_{k}}$ is the damping. The experiments have proved that the damping is not constant for every vibration mode but it depends on the quality factor which is different for every structure mode. The value of the quality vector is obtained by measurements on the structure for a given input voltage.

For the control matrix $B_{p}$, [4] or [11] can be consulted for the main steps regarding the modeling of the piezoelectric patches. As our experimental setup is not symmetric with respect to the $x$-axis - actuators only on one side of the plate - the position of the neutral fiber needs to be recalculated using [11]. The control matrix is then written as:

$$
B_{p}=\left(b_{p_{1}}, 0, \ldots, b_{p_{k}}, 0, \ldots, b_{p_{N}}, 0\right)^{T}
$$

where

$$
\begin{aligned}
b_{p_{k}}= & K_{b}\left(Y_{i}^{\prime}\left(y_{a 2}\right)-Y_{i}^{\prime}\left(y_{a 1}\right)\right) \int_{z_{a 1}}^{z_{a 2}} Z_{j}(z) d z \\
& +K_{b}\left(Z_{j}^{\prime}\left(z_{a 2}\right)-Z_{j}^{\prime}\left(z_{a 1}\right)\right) \int_{y_{a 1}}^{y_{a 2}} Y_{i}(y) d y
\end{aligned}
$$

$\left(y_{a 1}, z_{a 1}\right),\left(y_{a 2}, z_{a 2}\right)$ are the coordinates of the actuator and $K_{b}$ is a parameter depending on the actuator/plate geometry and on the position of the neutral fiber. In (10), $u(t)$ stands for the voltage applied to the piezoelectric patch. 
The output matrix $C_{p}$ is calculated with the new position of the neutral fiber:

$$
C_{p}=\left(0, c_{p_{1}}, \ldots, 0, c_{p_{k}}, \ldots, 0, c_{p_{N}}\right)
$$

where

$$
\begin{array}{r}
c_{p_{k}}=\frac{K_{c}}{\omega_{p_{k}} C_{a}}\left(\left(Y_{i}^{\prime}\left(y_{c 2}\right)-Y_{i}^{\prime}\left(y_{c 1}\right)\right) \int_{z_{c 1}}^{z_{c 2}} Z_{j}(z) d z\right. \\
\left.+\left(Z_{j}^{\prime}\left(z_{c 2}\right)-Z_{j}^{\prime}\left(z_{c 1}\right)\right) \int_{y_{c 1}}^{y_{c 2}} Y_{i}(y) d y\right)
\end{array}
$$

and $C_{a}$ is the capacity of the charge amplifier we use to measure the voltage in the output of the piezoelectric sensor and $K_{c}$ is a parameter depending on the sensor/plate geometry and on the position of the neutral fiber. The coordinates of the sensor patch are $\left(y_{c 1}, z_{c 1}\right),\left(y_{c 2}, z_{c 2}\right)$. For a more detailled calculation of the matrices $B_{p}$ and $C_{p}$ see [17].

2) Liquid model: In this section we calculate the finitedimensional approximation of the tank's sloshing.

Having the geometrical parameters of the rectangular tank one can use [12, Chapter 17] to calculate the movement equations and the boundary conditions. We then use the approximation given by [10] to model the sloshing. We are using a finite number of mass pendulums systems - each corresponding to a sloshing mode - that is to say for the $k^{t h}$ odd integer $i$ of the series (9), it corresponds a masspendulum system denoted $k$.

The state-space representation is then easily obtained using the pendulum equation under external acceleration:

$$
\ddot{z}_{k}+2 \xi_{z} \sqrt{\frac{g}{l_{k}}} \dot{z_{k}}+\frac{g}{l_{k}} z_{k}=-\frac{1}{l_{k}} C_{0}
$$

where $z_{k}$ is the angle of the $k$ th pendulum compared to its equilibrium position, $l_{k}$ its length and $\xi_{z}$ is the value of pendulum damping and it is fixed at 0.01 for all masspendulum systems.

By choosing the state space vector for the liquid sloshing equal to $X_{z}=\left(\begin{array}{ll}\dot{z_{k}} \sqrt{\frac{g}{l_{k}}} & z_{k}\end{array}\right)^{T}$, the dynamic equation is

$$
\dot{X}_{z}=A_{z} X_{z}+B_{z} u_{a c c}
$$

where the matrix $A_{z}$ computed from (14) is:

$$
A_{z}=\left(\begin{array}{cccc}
A_{z_{1}} & 0 & \cdots & 0 \\
0 & A_{z_{2}} & \cdots & 0 \\
& \cdots & & \\
0 & 0 & \cdots & A_{z_{N}}
\end{array}\right)
$$

with $A_{z_{k}}=\left(\begin{array}{cc}-2 \xi_{z} \sqrt{\frac{g}{l_{k}}} & -\sqrt{\frac{g}{l_{k}}} \\ \sqrt{\frac{g}{l_{k}}} & 0\end{array}\right)$.

In the case of the control matrix $B_{z}$ the construction is also direct by considering from (14) $u_{a c c}=C_{0}$ as the control variable:

$$
B_{z}=\left(b_{z_{1}}, 0, \ldots, b_{z_{k}}, 0, \ldots, b_{z_{N}}, 0\right)^{T}
$$

where $b_{z_{k}}=\left(\begin{array}{c}-\frac{1}{l_{k}} \\ 0\end{array}\right)$.
3) Model coupling: The crucial and the most difficult part in the modeling of this experimental setup is the interaction between the plate and the cylinder. In the following subsection the calculations are done assuming that the liquid movement is approximated by only one mass pendulum system and the plate movement by only one Ritz function. The generalization to more mass pendulum systems and more Ritz functions is direct.

In order to quantify the interaction between the plate and the tank we have to study the plate's influence on the tank and vice versa.

a) Plate's influence on the liquid filling the rectangular tank: The movement of the pendulum mass system under external acceleration is described by (14), where $C_{0}$ is the acceleration delivered by the movement of the plate. We first use (3) to express the movement of the plate and the acceleration is then obtained by derivations according to time. In the case of one mass-pendulum and one Ritz function the acceleration becomes:

$$
C_{0}=\ddot{w}(y, z, t)=Y_{1}(y) Z_{1}(z) \ddot{q}_{1}(t)
$$

where $Y_{1}(y)$ and $Z_{1}(z)$ are the first Ritz functions along $y$-axis and $z$-axis.

As in [10], the external acceleration $C_{0}$ can be calculated in the gravity center $G=\left(y_{G}, z_{G}\right)$ of the tank for steady motion. Thus (18) becomes:

$$
\ddot{w}\left(y_{G}, z_{G}, t\right)=Y_{1}\left(y_{G}\right) Z_{1}\left(z_{G}\right) \ddot{q}_{1}(t)=C_{0}
$$

and this allow us to write $C_{0}$ as:

$$
C_{0}=\left(\begin{array}{ll}
Y_{1}\left(y_{G}\right) Z_{1}\left(z_{G}\right) & 0
\end{array}\right)\left(\begin{array}{ll}
\ddot{q}_{1}(t) & \omega_{1} \dot{q}_{1}(t)
\end{array}\right)^{T} .
$$

From this last equation one can see $\left(\begin{array}{ll}\ddot{q}_{1}(t) & \omega_{1} \dot{q}_{1}(t)\end{array}\right)^{T}$ is the derivative of the state space vector of the rectangular plate $\dot{X}_{p}$. By taking $K_{G}=\left(\begin{array}{ll}Y_{1}\left(y_{G}\right) Z_{1}\left(z_{G}\right) & 0\end{array}\right)$, (19) becomes:

$$
C_{0}=K_{G} A_{p} X_{p}+K_{G} B_{p} u
$$

where $\dot{X}_{p}$ was replaced with the expression from (10).

Using (20), (16) and (17), equation (15) becomes:

$$
\dot{X}_{z}=A_{z} X_{z}+B_{z}\left(K_{G} A_{p} X_{p}+K_{G} B_{p} u\right)
$$

This equation describes the fact that the movement of the plate generates a movement of the pendulum mass systems, that is to say a movement of the liquid in the tank.

b) Tank's influence on the rectangular plate: The liquid sloshing is sensed by the plate as a perturbation that comes into the state space representation by means of a matrix $B_{z p}$ :

$$
\left\{\begin{array}{l}
\dot{X}_{p}=A_{p} X_{p}+B_{p} u+B_{z p} X_{z} \\
y=C_{p} X_{p}
\end{array}\right.
$$

see (paragraph III-B.1). For the calculation of the perturbation matrix $B_{z p}$ we are using the same approach as in the case of the control matrix $B_{p}$.

The external moment applied to the tank due to the movement of the fluid is computed in (9). By our choice of the physical parameters of the pendulum, the moment 
due to the liquid sloshing is equal to the moment due to the movement of all mass-pendulum systems. The external moment written for only one mass-pendulum is

$$
M_{z p}=m_{1} L_{1}\left(C_{0}+l_{1} \ddot{z}_{1}\right)
$$

where $C_{0}+l_{1} \ddot{z}_{1}$ is the total acceleration due to pendulum oscillations and plate movement. Using (20) and (21), the expression of $M_{z p}$ can be rewritten as:

$$
M_{z p}=\left(\begin{array}{ll}
m_{1} l_{1} L_{1} & 0
\end{array}\right)\left(\begin{array}{cc}
-2 \xi_{z} \sqrt{\frac{g}{l_{1}}} & -\sqrt{\frac{g}{l_{1}}} \\
\sqrt{\frac{g}{l_{1}}} & 0
\end{array}\right) X_{z}
$$

From this last equation we can infer that the total momentum of the pendulums does not depend on the external acceleration $C_{0}$ but only on the liquid movement.

For the calculation of $B_{z p}$ we are then assuming that the total momentum is concentrated in a small square around the tank center of gravity $G$, with edges of coordinates $\left(y_{1 G}, z_{1 G}\right),\left(y_{2 G}, z_{2 G}\right)$. The total bending moment is:

$$
\Gamma_{z p}=\int_{y_{1 G}}^{y_{2 G}} \int_{z_{1 G}}^{z_{2 G}}\left(\frac{\partial^{2} M_{z p}}{\partial y^{2}}+\frac{\partial^{2} M_{z p}}{\partial z^{2}}\right) w d y d z .
$$

Using (23) and (3) into (24) we obtain

$$
B_{z p}=\left(\begin{array}{c}
b_{z p_{1}} \\
0
\end{array}\right)
$$

where:

$$
\begin{aligned}
b_{z p_{1}}= & K_{z p}\left(\left(Y_{i}^{\prime}\left(y_{2 G}\right)-Y_{i}^{\prime}\left(y_{1 G}\right)\right) \int_{z_{1 G}}^{z_{2 G}} Z_{j}(z) d z\right. \\
& \left.+\left(Z_{j}^{\prime}\left(z_{2 G}\right)-Z_{j}^{\prime}\left(z_{1 G}\right)\right) \int_{y_{1 G}}^{y_{2 G}} Y_{i}(y) d y\right)
\end{aligned}
$$

and $K_{z p}=\left(\begin{array}{ll}m_{1} l_{1} L_{1} & 0\end{array}\right) A_{z}$.

\section{Complete Model}

From (21) and (22) one can write the complete state-space model of the experimental setup. For the sake of clarity of the presentation of the complete model we consider the case of one Ritz function and one mass-pendulum.

For the complete system we choose as state space vector:

$$
X=\left(\begin{array}{c}
X_{p} \\
X_{z}
\end{array}\right)=\left(\begin{array}{llll}
\dot{q}_{1} & \omega_{1} q_{1} & \dot{z}_{1} & \sqrt{\frac{g}{l_{1}}} z_{1}
\end{array}\right)^{T} .
$$

Combining all the matrices leads to the following finitedimensional approximation using the state-space representation:

$$
\left\{\begin{array}{l}
\dot{X}=\left(\begin{array}{cc}
A_{p} & B_{z p} \\
A_{p z} & A_{z}
\end{array}\right) X+\left(\begin{array}{c}
B_{p} \\
B_{p z}
\end{array}\right) u \\
y=\left(\begin{array}{cc}
C_{p} & \mathbf{0}
\end{array}\right) X
\end{array}\right.
$$

where $A_{p z}=\left(\begin{array}{c}-\frac{1}{l_{1}} K_{G} A_{p} \\ \mathbf{0}\end{array}\right), B_{p z}=\left(\begin{array}{c}-\frac{1}{l_{1}} K_{G} B_{p} \\ \mathbf{0}\end{array}\right)$ and 0 denotes the null matrix of appropriate dimensions.

\section{CONTROLler SYNTHESIS}

In this section we calculate a controller for our experimental setup using the finite-dimensional approximation of our model.

Using the state space representation (26) we can use the pole allocation method to compute a controller $K$ that will minimize the response time of the closed-loop in comparison to the open-loop.

The controller $K$ is first tested in simulation under Matlab and then on the experimental setup at ISAE. In our case the voltage delivered by $K$ will be the input of the system and the voltage delivered by the piezoelectric sensor will be the system's output. The tank is filled up to an arbitrary level of 0.7 . The initial condition considered is a plate displacement of $2 \mathrm{~cm}$ from its initial equilibrium position. In choosing the closed-loop poles, we have to take into account the limitations of the piezoelectric actuators $[-130 \mathrm{~V}, 130 \mathrm{~V}]$.

\section{A. Simulations}

The controller is calculated using the first three modes of the plate and the two modes of the liquid sloshing, that is to say (26) is used with $X_{p} \in \mathbb{R}^{6}$ and $X_{z} \in \mathbb{R}^{4}$.

The closed-loop system is then simulated using the first four modes of the plate and the first three modes of the liquid sloshing (therefore it is simulated on a system of larger dimension). The voltage delivered by the piezoelectric sensors in the open-loop and the closed-loop case are presented in Figure 3. The real part of the closed-loop poles was chosen up to 10 times the real part of the open-loop ones. The state estimator is a Luenberger observer with the same dimension as the system. The real part of the observer poles is up to 10 times bigger than the real part of the poles.

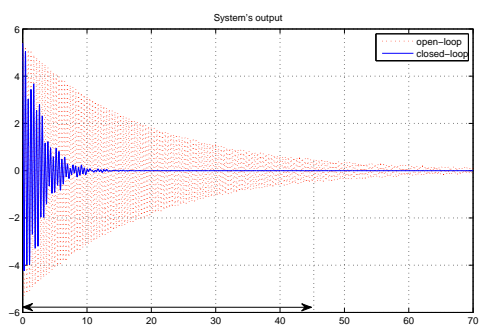

Fig. 3. Response of the closed-loop system (in plain line) and of the open-loop system (in dotted line) on numerical simulations

The voltage delivered by the pole placement controller is presented in Figure 4. The voltage being in the range $[-130 \mathrm{~V}, 130 \mathrm{~V}]$, the most important constraint is respected.

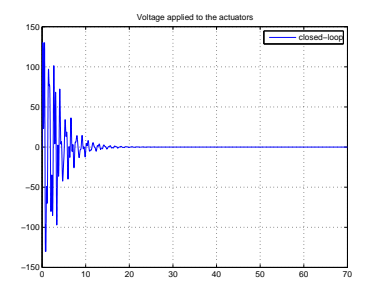

Fig. 4. Voltage applied to the actuators on numerical simulations 
From Figure 3 one can notice the absence of the spillover effect on the fourth mode (i.e. the first one which has been neglected in the design on the controller). Although it is a common problem when working with infinite-dimensional systems we did not observe it. Even though some techniques to cancel it exist, e.g. by prefiltering the piezoelectric sensor signals with a low-pas filter, see [8].

\section{B. Experiments}

The results are then tested on the experimental setup. The controller previously calculated is implemented under the same initial conditions (plate displacement of $2 \mathrm{~cm}$ from its initial position). The voltage delivered by the system's sensors, in the open-loop and the closed-loop case, is given in Figure 5. The response time (settling time) is measured considering a $10 \%$ tolerance band. We can see that the closed-loop response is better that the open-loop one, even though we considered only a few modes for the plate/liquid.

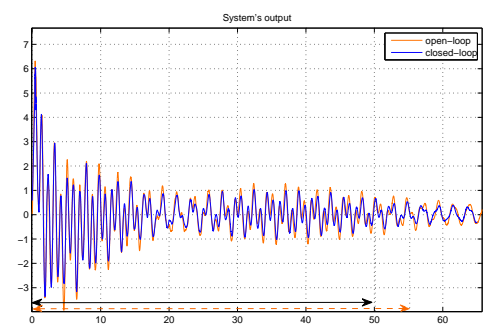

Fig. 5. Response of the closed-loop system (in plain line) and of the open-loop system (in dotted line) on experiments

Figure 6 gives the voltage delivered by the pole placement controller on the experimental setup.

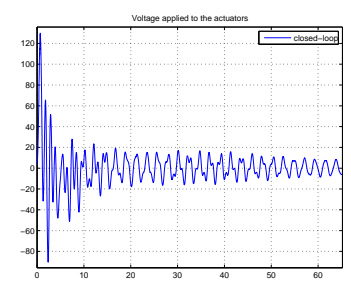

Fig. 6. Voltage applied to the actuators on experiments

By considering Figures 3 and 5 one can notice an openloop response time of 54 seconds during experimental tests and of 45 seconds during numerical simulations. Because of the closeness of the response time we infer the validity of our approximation and of our model.

\section{CONCLUSION}

After settling an infinite-dimensional model we have computed a finite-dimension approximation for the experimental setup by using Ritz method. Tests and simulations are made with only three modes for the plate and two modes for the liquid. Due to the closeness in open-loop response time between the model and the experimental setup we infer the validity of the model. However the response time of the closed loop systems is not so close in both cases. It is probably due to the uncertainty that was not taken into consideration while calculating the controller.

Further investigations are necessary in some areas and are studied now such as:

1. Damping modeling. We considered the damping of the structure being constant for each mode. In reality we observed that the damping depends not only on the mode but also on the input voltage of the structure.

2. $H_{\infty}$ controller. Constructed with a large number of plate/liquid modes, it will make the system robust to exterior perturbations. Also the use of uncertainty in modeling the system and in the synthesis of the controller will improve the closeness between the closed loop response time.

3. Non linear modeling of the actuators.

\section{ACKNOWLEDGEMENTS}

The authors would like to thank Valérie PommierBudinger, ISAE, Toulouse (France) for fruitful discussions on the experimental setup.

\section{REFERENCES}

[1] B. Bhikkaji, S. O. R. Moheimani, and I. R. Petersen. Multivariable integral control of resonant structures. 47th IEEE Conference on Decision and Control 2008, pages 3743-3748, 9-11 Dec. 2008.

[2] R. D. Blevins. Formulas for natural frequency and mode shape. Krieger publishing company, Florida, 1995.

[3] E. Crépeau and C. Prieur. Control of a clamped-free beam by a piezoelectric actuator. ESAIM: Control, Optim. Cal. Var., 12:545-563, 2006.

[4] E.K. Dimitriadis, C.R. Fuller, and C.A. Rogers. Piezoelectric actuators for distributed vibration excitation of thin plates. Journal of Vibrational Acoustics, 113:100-107, 1991.

[5] F.T. Dodge. The new "dynamic behavior of liquids in moving containers". Technical report, Southwest Research Institute, San Antonio, Texas, 2000.

[6] A.J. Fleming and S.O. Reza Moheimani. Optimal impedance design for piezoelectric vibration control. Conference on Decision and Control 2004, 3:2596 - 2601, 14-17 Dec. 2004.

[7] M. Géradin and D. Rixen. Mechanical Vibrations: theory and application to structural dynamics. Masson, 1994.

[8] J.K. Hwang, C.-H. Choi, C.K. Song, and J.M. Lee. Robust LQG control of an all-clamped thin plate with piezoelectric actuators/sensors. IEEE/ASME, 2:205-212, september 1997.

[9] R. A. Ibrahim. Liquid sloshing dynamics. Cambridge Univ. Press, 2005.

[10] R.S. Khandelwal and N.C. Nigam. A mechanical model for liquid sloshing in a rectangular container. Journal of the institution of engineers, India, 69:152-156, 1989.

[11] S.J. Kim and J.D. Jones. Influence of piezo-actuator thickness on the active vibration control of a cantilever beam. Journal of Intellingent Material Systems and Structures, 6:610-623, 1995.

[12] Sir H. Lamb. Hydrodynamics. Cambridge Mathematical Library, 1995.

[13] I. Lasiecka and A. Tuffaha. Boundary feedback control in fluidstructure interactions. 47th IEEE Conference on Decision and Control, 2008, pages 203 - 208, 9-11 Dec. 2008.

[14] Y. K. Lee and D. Halim. Vibration control experiments on a piezoelectric laminate plate using spatial feedforward control approach. 43rd IEEE Conference on Decision and Control, 2004, pages 2403 - 2408, 14-17 Dec. 2004.

[15] V. Pommier-Budinger, Y. Janat, D. Nelson-Gruel, P. Lanusse, and A. Oustaloup. Fractional robust control with iso-damping property. American Control Conference, pages 4954 - 4959, 11-13 June 2008.

[16] T. Terasawa, C. Sakai, H. Ohmori, and A. Sano. Adaptive identification of MR damper for vibration control. 43rd IEEE Conference on Decision and Control, 2004, 3:2297 - 2303, 14-17 Dec. 2004.

[17] S. Tliba, H. Abou-Kandil, and C. Prieur. Active vibration damping of a smart flexible structure using piezoelectric transducers: $H_{\infty}$ design and experimental results. IFAC World Congress on Automatic Control, 2005, Prague, Czech Republic, 4-8 July 2005. 